\title{
Design, Synthesis and Molecular Docking of some Oxazolidinone Compounds
}

\author{
LUCIA PINTILIE ${ }^{1 *}$, AMALIA STEFANIU ${ }^{1}$, ALINA IOANA NICU르, CATALINA NEGUT ${ }^{1}$, CONSTANTIN TANASE ${ }^{1}$, \\ MIRON TEODOR CAPROIU ${ }^{2}$ \\ ${ }^{1}$ National Institute for Chemical-Pharmaceutical Research and Development, 112 Vitan Av., 74373, Bucharest, Romania \\ ${ }^{2}$ Organic Chemistry Center C.D.Nenitescu, 202 B Splaiul Independentei, Bucharest, Romania
}

\begin{abstract}
A series of oxazolidinone compounds have been obtained and characterized by physico-chemical methods and antimicrobial activity against Staphylococcus Aureus ATCC 6538. For the synthesized compounds have been performed calculations of characteristics and molecular properties, using Spartan 14 Software from Wavefunction, Inc. Irvine, CA. and molecular docking studies using CLC Drug Discovery Workbench 2.4 software, to identify and visualize the most likely interaction ligand (oxazolidinone derivatives) with the receptor protein.
\end{abstract}

Keywords: linezolid, oxazolidinone, antimicrobial activity, molecular docking

Infectious diseases are the second leading cause of death worldwide [1]. In this context, newer drugs have been developed. Linezolid (Figure 1) is a synthetic drug belonging to oxazolidinone class that was approved by the FDA in 1999. This new drug shows a great activity against gram-positive microorganisms, especially methicilin resistant St. Aureus (MRSA), methicilin resistant St. Epidermis (MRSE) and Vancomicyn Resistant Enterococci (VRE) [2-7].

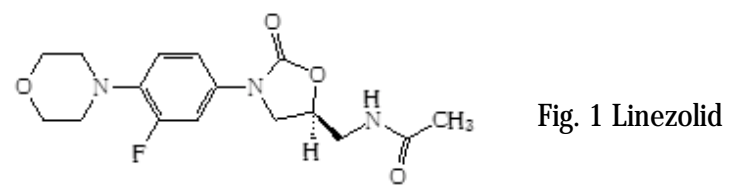

\section{Experimental part}

Melting points were determined in opened capillary on Melting point apparatus OptiMelt and are uncorrected. Progress of the reaction was followed by TLG on Merck silica gel $60 \mathrm{~F}_{254}$ plates eluted with the solvent system: tetrahydrofuran:dioxan: ammoniac (60:20:30) (v:v:v). ${ }^{1} \mathrm{H}$ and ${ }^{13}$ C-NMR spectra were recorded in $\mathrm{CDCl}_{3}$, DMSO-d and trifluoroacetic acid, on two instruments Varian, Varian Gemini 300 BB (operating at $300 \mathrm{MHz}$ for proton and 75 $\mathrm{MHz}$ for carbon) and UNITY 400 Plus(operating at $400 \mathrm{MHz}$ for proton and $100 \mathrm{MHz}$ for carbon). Tetramerthylsilane as internal standard was the reference for the chemical shifts. All chemical shifts are given in the delta scale (ppm vs internal TMS). FT IR was recorded on an instrument Bruker Vertex 70 with diamond optic. UV-Vis was recorded on an instrument UV - Vis LAMBDA 12. Elemental analysis was

Synthesis of 1-(2-fluoro-4-nitrophenyl)-3-methyl-piperidine A solution of $0.03 \mathrm{Mol}(5 \mathrm{~g})$ 3,4-difluoronitrobenzene in $50 \mathrm{~mL}$ methanol, was treated with $0.075 \mathrm{~mol}(7.15 \mathrm{~g}) 3-$ methyl-piperidine, followed by stirring $5 \mathrm{~h}$ at $50^{\circ}$. The solution was cooled to room temperature and concentrated in vacuum. The residue was dissolved in 50 $\mathrm{mL}$ ethyl acetate and extracted with $2 \times 50 \mathrm{~mL}$ water and $50 \mathrm{~mL}$ saturated sodium chloride solution followed by drying on sodium sulfate. The solution was concentrated in vacuum to afford an brown oil which was chromatographed over $200 \mathrm{~g}$ silica gel and eluting with toluene-ethyl acetate : 1:1 (v:v) to yield $6.43 \mathrm{~g}$ 1-(2-fluoro4-nitrophenyl)-3-methyl-piperidine (oil, yield. $90 \%$ ). ${ }^{1 \mathrm{H}}$ $\mathrm{NMR}\left(\mathrm{CDCl}_{3}\right.$, ä ppm, J Hz): 7.94(dd, $1 \mathrm{H}, \mathrm{H}-6, J\left(\mathrm{~F}-\mathrm{H}^{6}\right)=2.2$, $\left.{ }^{4}\left(\mathrm{H}^{5}-\mathrm{H}^{6}\right)=8.8\right) ; 7.85\left(\mathrm{dd}, 1 \mathrm{H}, \mathrm{H}-2,{ }^{4}\left(\mathrm{H}^{2}-\mathrm{H}^{6}\right)=2.5,4 J(\mathrm{~F}-\right.$ $\left.\left.\mathrm{H}^{2}\right)=13.2\right) ; 6.90\left(\mathrm{t}, 1 \mathrm{H}, \mathrm{H}-5, J\left(\mathrm{~F}-\mathrm{H}^{5}\right)=3\left(\mathrm{H}^{5}-\mathrm{H}^{6}\right)=8.8\right)$; $3.27\left(\mathrm{~m}, 4 \mathrm{H}, 2 \mathrm{H}-7,2 \mathrm{H}-11\right.$, syst. A $\left.\mathrm{B}_{2}\right) ; 1.60 .1 .80(\mathrm{~m}, 6 \mathrm{H}, 2 \mathrm{H}-$ 8, 2H-9, 2H-10). ${ }^{13} \mathrm{C}-\mathrm{NMR}\left(\mathrm{CDCl}_{3^{\prime}}{ }^{2} \delta \mathrm{ppm}\right): 152.77(\mathrm{~d}, \mathrm{C}-3$, $\left.J\left(\mathrm{~F}^{\left.-\mathrm{C}^{3}\right)}=248.5 \mathrm{~Hz}\right) ; 146.46\left(\mathrm{~d}^{3} \mathrm{C}-1,3\right)\left(\mathrm{F}-\mathrm{C}^{1}\right)=7.4 \mathrm{~Hz}\right)$; 139.56(d, C-4, J(F-C $\left.\left.\mathrm{C}^{4}\right)=8.0 \mathrm{~Hz}\right) ; 121.06\left(\mathrm{~d}, \mathrm{C}-5,3\left(\mathrm{~F}-\mathrm{C}^{5}\right)=2.9\right.$ $\left.\mathrm{Hz}) ; 116.38(\mathrm{~d}, \mathrm{C}-6,4)\left(\mathrm{F}-\mathrm{C}^{6}\right)=4.3 \mathrm{~Hz}\right) ; 112.32(\mathrm{~d}, \mathrm{C}-2, J(\mathrm{~F}-$ $\left.\left.\mathrm{C}^{2}\right)=26.6 \mathrm{~Hz}\right) ; 51.00\left(\mathrm{~d}, \mathrm{C}-7, \mathrm{C}-11,4\left(\mathrm{~F}-\mathrm{C}^{7,11}\right)=5.2 \mathrm{~Hz}\right)$; 25.81(C-8, C-10); 24.11(C-9).

Synthesis of 1-(2-fluoro-4-nitrophenyl)-4-methylpiperidine

1-(2-fluoro-4-nitrophenyl)-4-methyl-piperidine: (oil, yield. 85\%). ${ }^{1} \mathrm{H}-\mathrm{NMR}\left(\mathrm{CDCl}_{3}, \delta \mathrm{ppm}, J \mathrm{~Hz}\right): 7.94(\mathrm{dd}, 1 \mathrm{H}, \mathrm{H}-6$, $\left.J\left(\mathrm{~F}-\mathrm{H}^{6}\right)=2.2,4\left(\mathrm{H}^{5}-\mathrm{H}^{6}\right)=8.8\right) ; 7.85\left(\mathrm{dd}, 1 \mathrm{H}, \mathrm{H}^{-} 2,{ }^{\prime}\right)\left(\mathrm{H}^{2}-\right.$ $\left.\left.\mathrm{H}^{6}\right)=2.5,4 J\left(\mathrm{~F}-\mathrm{H}^{2}\right)=13.2\right) ; 6.90\left(\mathrm{t}, 1 \mathrm{H}, \mathrm{H}-5, J\left(\mathrm{~F}-\mathrm{H}^{5}\right)=3 J\left(\mathrm{H}^{5}\right.\right.$ $\left.\left.\mathrm{H}^{6}\right)=8.8\right) ; 3.88(\mathrm{~m}, 4 \mathrm{H}, \mathrm{H}-7, \mathrm{H}-11) ; 2.87(\mathrm{~m}, 2 \mathrm{H}, \mathrm{H}-7, \mathrm{H}-11)$; $1.77(\mathrm{~m}, 2 \mathrm{H}, \mathrm{H}-8, \mathrm{H}-10) ; 1.60(\mathrm{~m}, 1 \mathrm{H}, \mathrm{H}-9) ; 1.39(\mathrm{~m}, 2 \mathrm{H}, \mathrm{H}-8$, $\mathrm{H}-10) ; 1.00(\mathrm{~d}, 3 \mathrm{H}, \mathrm{H}-12,6.6) .{ }^{13} \mathrm{C}-\mathrm{NMR}\left(\mathrm{CDCl}{ }_{3}, \delta \mathrm{ppm}\right)$ : 152.75(d, C-3, J(F-C 3$)=247.8 \mathrm{~Hz}) ; 146.26\left(\mathrm{~d}^{3}, \mathrm{C}-1,3\right)(\mathrm{F}-$ $\left.\left.\mathrm{C}^{1}\right)=7.9 \mathrm{~Hz}\right) ; 139.66\left(\mathrm{~d}, \mathrm{C}-4, J\left(\mathrm{~F}-\mathrm{C}^{4}\right)=8.9 \mathrm{~Hz}\right) ; 121.05(\mathrm{~d}, \mathrm{C}-5$, $\left.{ }^{3}\left(\mathrm{~F}-\mathrm{C}^{5}\right)=2.9 \mathrm{~Hz}\right) ; 117.06\left(\mathrm{~d}, \mathrm{C}-6,{ }^{4}\left(\mathrm{~F}-\mathrm{C}^{6}\right)=4.5 \mathrm{~Hz}\right) ; 112.50(\mathrm{~d}$, $\mathrm{C}-2$, J $\left.\left(\mathrm{F}-\mathrm{C}^{2}\right)=27.8 \mathrm{~Hz}\right) ; 50.34\left(\mathrm{~d}, \mathrm{C}-7, \mathrm{C}-11, J\left(\mathrm{~F}-\mathrm{C}^{7}\right)=5.1 \mathrm{~Hz}\right)$; 34.02(C-8, C-10); 31.65(C-9); 21.79(C-12).

Synthesis of 1-(2-fluoro-4-nitrophenyl)-piperidine

1-(2-fluoro-4-nitrophenyl)-piperidine: (oil, yield. 93\%). ${ }^{1} \mathrm{H}-\mathrm{NMR}\left(\mathrm{CDCl}_{3^{\prime}} \delta \mathrm{ppm}, J \mathrm{~Hz}\right): 7.94\left(\mathrm{dd}, 1 \mathrm{H}, \mathrm{H}-6, J\left(\mathrm{~F}-\mathrm{H}^{6}\right)=\right.$ 2.2, $\left.\left.{ }^{4}\left(\mathrm{H}^{5}-\mathrm{H}^{6}\right)=8.8\right) ; 7.85\left(\mathrm{dd}, 1 \mathrm{H}, \mathrm{H}-2,{ }^{4}\right)\left(\mathrm{H}^{2}-\mathrm{H}^{6}\right)=2.5,4\right)(\mathrm{F}-$ $\left.\left.H^{2}\right)=13.2\right) ; 6.90\left(t, 1 H, H-5, J\left(F-H^{5}\right)=3 J\left(H^{5}-H^{6}\right)=8.8\right)$; $3.27\left(\mathrm{~m}, 4 \mathrm{H}, 2 \mathrm{H}-7,2 \mathrm{H}-11\right.$, syst. $\left.\mathrm{A}_{2} \mathrm{~B}_{2}\right) ; 1.60 .1 .80(\mathrm{~m}, 6 \mathrm{H}, 2 \mathrm{H}-$ 8, 2H-9, 2H-10). ${ }^{13} \mathrm{C}-\mathrm{NMR}\left(\mathrm{CDCl}_{3}, \delta \mathrm{ppm}\right): 152.77(\mathrm{~d}, \mathrm{C}-3$, $\left.\left.J\left(\mathrm{~F}^{-} \mathrm{C}^{3}\right)=248.5 \mathrm{~Hz}\right) ; 146.46(\mathrm{~d}, \mathrm{C}-1,3)\left(\mathrm{F}-\mathrm{C}^{1}\right)=7.4 \mathrm{~Hz}\right)$; 139.56(d, C-4, J(F-C $\left.)^{4}=8.0 \mathrm{~Hz}\right) ; 121.06\left(\mathrm{~d}, \mathrm{C}-5,3\left(\mathrm{~F}-\mathrm{C}^{5}\right)=2.9\right.$ $\left.\mathrm{Hz}) ; 116.38(\mathrm{~d}, \mathrm{C}-6,4)\left(\mathrm{F}-\mathrm{C}^{6}\right)=4.3 \mathrm{~Hz}\right) ; 112.32(\mathrm{~d}, \mathrm{C}-2$, J(F$\left.\left.\mathrm{C}^{2}\right)=26.6 \mathrm{~Hz}\right) ; 51.00\left(\mathrm{~d}, \mathrm{C}-7, \mathrm{C}-11,4 \mathrm{~J}\left(\mathrm{~F}-\mathrm{C}^{7,11}\right)=5.2 \mathrm{~Hz}\right)$; 25.81(C-8, C-10); 24.11(C-9).

Synthesis of 1-(2-fluoro-4-nitrophenyl)-morpholine

1-(2-fluoro-4-nitrophenyl)-morfoline: (m.p. $108-110.9^{\circ} \mathrm{C}$, yield 94\%). ${ }^{1} \mathrm{H}-\mathrm{NMR}\left(\mathrm{CDCl}_{3,} \delta \mathrm{ppm}, J \mathrm{~Hz}\right): 7.99(\mathrm{dd}, 1 \mathrm{H}, \mathrm{H}-6$, $\left.{ }_{4}^{4}\left(\mathrm{~F}-\mathrm{H}^{6}\right)=2.2,{ }^{4}\left(\mathrm{H}^{5}-\mathrm{H}^{6}\right) \stackrel{3^{\prime}}{=} 8.8\right) ; 7.91\left(\mathrm{dd}, 1 \mathrm{H}, \mathrm{H}-2,{ }^{4}\right)\left(\mathrm{H}^{2}-\right.$

*email:Iucia.pintilie@gmail.com; Phone: (+40)723619187 
$\left.\left.H^{6}\right)=2.5, J\left(F-H^{2}\right)=13.2\right) ; 6.93\left(t, 1 H, H-5,5\left(F-H^{5}\right)=3\left(H^{5}\right.\right.$ $\left.\left.H^{6}\right)=8.8\right) ; 3.88\left(m, 4 H, H-8, H-10\right.$, syst. $\left.A_{2} B_{2}\right) ; 3.29(m, 4 H$, $\mathrm{H}-7, \mathrm{H}-11$, syst. $\left.\mathrm{A}, \mathrm{B}_{2}\right) .{ }^{13} \mathrm{C}-\mathrm{NMR}\left(\mathrm{CDCl}{ }_{3}, \delta \mathrm{ppm}^{2}\right): 153.12(\mathrm{~d}, \mathrm{C}-$ 3, $\left.J\left(\mathrm{~F}^{-\mathrm{C}^{3}}\right)=249.7 \mathrm{~Hz}\right) ; 145.49\left(\mathrm{~d}^{3}, \mathrm{C}-1,3 /\left(\mathrm{F}-\mathrm{C}^{1}\right)=7.6\right.$ $\mathrm{Hz}) ; 140.86\left(\mathrm{~d}, \mathrm{C}-4, J\left(\mathrm{~F}-\mathrm{C}^{4}\right)=9.8 \mathrm{~Hz}\right) ; 120.99(\mathrm{~d}, \mathrm{C}-5,3)(\mathrm{F}-$ $\left.\left.\left.\mathrm{C}^{5}\right)=2.9 \mathrm{~Hz}\right) ; 116.88(\mathrm{~d}, \mathrm{C}-6,4)\left(\mathrm{F}-\mathrm{C}^{6}\right)=4.0 \mathrm{~Hz}\right) ; 112.61(\mathrm{~d}, \mathrm{C}-$ 2, J(F-C $\left.\left.{ }^{2}\right)=26.4 \mathrm{~Hz}\right) ; 66.58(\mathrm{C}-8, \mathrm{C}-10)$; 49.89(d, C-7, C-11, $\left.J\left(\mathrm{~F}-\mathrm{C}^{7,11}\right)=5.2 \mathrm{~Hz}\right)$.

Synthesis of 3-fluoro-4-(3-methyl-piperidinyl)-aniline

A solution of $0.03 \mathrm{Mol}$ (7.12 g) 1-(2-fluoro-4-nitrophenyl)3-methyl-piperidine in $100 \mathrm{~mL}$ acetone, was treated with $0.09 \mathrm{~mol}(5.7 \mathrm{~g})$ ammonium formate $(6.53 \mathrm{~g})$ and 0.0712 $\mathrm{g} \mathrm{Pd} / \mathrm{C}$ followed by stirring $6 \mathrm{~h}$ at $50^{\circ}$. The solution was cooled to room temperature and was filtered. The filtrate was concentrated in vacuo. The residue was dissolved in $50 \mathrm{~mL}$ ethyl acetate and extracted with $2 \times 50 \mathrm{~mL}$ water and $50 \mathrm{~mL}$ saturated sodium chloride solution followed by drying on sodium sulfate. The solution was concentrated in vacuum to afford an brown oil which was chromatographed over $200 \mathrm{~g}$ silica gel and eluting with toluene-ethyl acetate: 3:1 (v:v) to yield $5 \mathrm{~g}$ 3-fluoro-4-(3methyl-piperidinyl)-aniline (oil, yield. $80 \%$ ). ${ }^{1} \mathrm{H}-\mathrm{NMR}\left(\mathrm{CDCl}_{3}\right.$, ä ppm, J Hz): 6.81(t, $\left.1 \mathrm{H}, \mathrm{H}-5,{ }^{4} J\left(\mathrm{~F}-\mathrm{H}^{5}\right)=3\left(\mathrm{H}^{5}-\mathrm{H}^{6}\right)=8.9\right)^{3}$; $6.45 \div 6.35(\mathrm{~m}, 2 \mathrm{H}, \mathrm{H}-2, \mathrm{H}-6) ; 3.51\left(\mathrm{bs}, 2 \mathrm{H}, \mathrm{NH}_{2}\right) ; 3.18(\mathrm{~m}$, $4 \mathrm{H}, 2 \mathrm{H}-7,2 \mathrm{H}-11) ; 2.44(\mathrm{~m}, 1 \mathrm{H}, \mathrm{H}-8) ; 2.19(\mathrm{t}, 1 \mathrm{H}, \mathrm{H}-9)$; $1.83 \div 1.75(\mathrm{~m}, 3 \mathrm{H}, \mathrm{H}-9,2 \mathrm{H}-10) ; 0.92(\mathrm{~d}, 3 \mathrm{H}, \mathrm{H}-12,6.5) .{ }^{13} \mathrm{C}-$ $\operatorname{NMR}\left(C D C l_{2}, \delta \mathrm{ppm}\right): 156.72\left(\mathrm{~d}, \mathrm{C}-3, J\left(\mathrm{~F}-\mathrm{C}^{3}\right)=244.7\right.$ $\mathrm{Hz}) ; 142.21\left(\mathrm{~d}, \mathrm{C}-1,3 /\left(\mathrm{F}-\mathrm{C}^{1}\right)=10.3 \mathrm{~Hz}\right) ; 133.10(\mathrm{~d}, \mathrm{C}-4$, J(F$\left.\left.\mathrm{C}^{4}\right)=10.3 \mathrm{~Hz}\right) ; 120.58\left(\mathrm{~d}, \mathrm{C}-5,3\left(\mathrm{~F}-\mathrm{C}^{5}\right)=4.2 \mathrm{~Hz}\right) ; 110.56(\mathrm{~d}, \mathrm{C}-$ $\left.6,{ }^{4} J\left(F-C^{6}\right)=2.9 \mathrm{~Hz}\right) ; 103.87\left(\mathrm{~d}, \mathrm{C}-2, J\left(\mathrm{~F}-\mathrm{C}^{2}\right)=24.4 \mathrm{~Hz}\right)$; $60.17\left(\mathrm{~d}, \mathrm{C}-7\right.$ or $\left.\mathrm{C}-11, \mathrm{~J}\left(\mathrm{~F}-\mathrm{C}^{7}\right)=2.5 \mathrm{~Hz}\right) ; 52.37(\mathrm{~d}, \mathrm{C}-7$ or $\mathrm{C}-11$, $\left.J\left(\mathrm{~F}^{-} \mathrm{C}^{7}\right)=2.3 \mathrm{~Hz}\right) ; 32.81(\mathrm{C}-9$ or $\mathrm{C}-10) ; 31.43(\mathrm{C}-8) ; 25.87(\mathrm{C}-$ 9 or C-10); 19.56(C-12).

Synthesis of 3-fluoro-4-(4-methyl-piperidinyl)-aniline

3-fluoro-4-(4-methyl-piperidinyl)-aniline: (oil, yield. 79\%). ${ }^{1} \mathrm{H}-\mathrm{NMR}\left(\mathrm{CDCl}_{3,} \delta \mathrm{ppm}, J \mathrm{~Hz}\right): 6.81(\mathrm{t}, 1 \mathrm{H}, \mathrm{H}-5,4 J(\mathrm{~F}-$ $\left.\left.\mathrm{H}^{5}\right)=3\left(\mathrm{H}^{5}-\mathrm{H}^{6}\right)=9.1\right) ; 6.44 \div 6.35(\mathrm{~m}, 2 \mathrm{H}, \mathrm{H}-2, \mathrm{H}-6) ; 3.50(\mathrm{bs}$, $2 \mathrm{H}, \mathrm{NH}_{2}$, deuterable); $3.23(\mathrm{~m}, 2 \mathrm{H}, \mathrm{H}-7, \mathrm{H}-11$ or $\mathrm{H}-7, \mathrm{H}-$

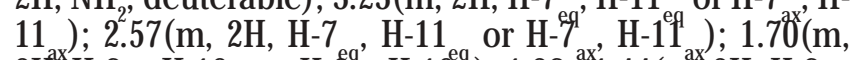
$2 \mathrm{H}^{2 x} \mathrm{H}-8, \mathrm{H}-10$ or $\left.\mathrm{H}-8^{q^{\prime}}, \mathrm{H}-10^{\mathrm{eq}}\right) ; 1.38 \div 1.44(\mathrm{~m}, 3 \mathrm{H}, \mathrm{H}-8$

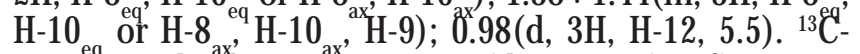

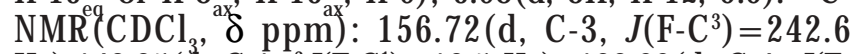
$\mathrm{Hz}$ ); 142.25 (d, C-1, 3 (F-C $\left.\left.\mathrm{C}^{1}\right)=10.5 \mathrm{~Hz}\right) ; 132.99(\mathrm{~d}, \mathrm{C}-4, J(\mathrm{~F}-$ $\left.\left.C^{4}\right)=10.0 \mathrm{~Hz}\right) ; 120.52\left(\mathrm{~d}, \mathrm{C}-5,3\left(\mathrm{~F}^{-C^{5}}\right)=4.4 \mathrm{~Hz}\right) ; 110.56(\mathrm{~d}$, $\left.\mathrm{C}-6,4 J\left(\mathrm{~F}-\mathrm{C}^{6}\right)=2.9 \mathrm{~Hz}\right) ; 103.38\left(\mathrm{~d}, \mathrm{C}-2, J\left(\mathrm{~F}-\mathrm{C}^{2}\right)=22.5\right.$ $\mathrm{Hz}) ; 52.31\left(\mathrm{~d}, \mathrm{C}-7, \mathrm{C}-11, j\left(\mathrm{~F}-\mathrm{C}^{7,11}\right)=2.0 \mathrm{~Hz}\right) ; 34.61(\mathrm{C}-8, \mathrm{C}-$ 10); 30.57(C-9); 11.97(C-12).

\section{Synthesis of 3-fluoro-4-piperidinyl-aniline}

3-fluoro-4-piperidinyl-aniline: (oil, yield. 92.7\%). ${ }^{1 \mathrm{H}}$ $\operatorname{NMR}\left(\mathrm{CDCl}_{3}, \delta \mathrm{ppm}, J \mathrm{~Hz}\right): 6.81\left(\mathrm{t}, \mathrm{H}, \mathrm{H}-5,{ }^{4}\left(\mathrm{~F}-\mathrm{H}^{5}\right)=3\left(\mathrm{H}^{5}\right.\right.$ $\left.\left.\mathrm{H}^{6}\right)=9.2\right) ; 6.44 \div 6.35(\mathrm{~m}, 2 \mathrm{H}, \mathrm{H}-2, \mathrm{H}-6) ; 3.51\left(\mathrm{bs}, 2 \mathrm{H}, \mathrm{NH}_{3}\right.$ deuterable); $2.89(\mathrm{t}, 4 \mathrm{H}, \mathrm{H}-7, \mathrm{H}-11,5.5) ; 1.71(\mathrm{qv}, 4 \mathrm{H}, \mathrm{H}-8$, $\mathrm{H}-10,5.5) ; 1.52(\mathrm{~m}, 2 \mathrm{H}, \mathrm{H}-9) .{ }^{13} \mathrm{C}-\mathrm{NMR}\left(\mathrm{CDCl}_{3}, \delta \mathrm{ppm}\right)$ : $156.73\left(\mathrm{~d}, \mathrm{C}-3, J\left(\mathrm{~F}-\mathrm{C}^{3}\right)=242.5 \mathrm{~Hz}\right) ; 142.25\left(\mathrm{~d}^{3^{\prime}} \mathrm{C}-1,3\right)(\mathrm{F}-$ $\left.\left.\mathrm{C}^{1}\right)=10.2 \mathrm{~Hz}\right) ; 133.24\left(\mathrm{~d}, \mathrm{C}-4, J\left(\mathrm{~F}-\mathrm{C}^{4}\right)=9.5 \mathrm{~Hz}\right) ; 120.51(\mathrm{~d}, \mathrm{C}-$ $\left.5,3\left(F^{2}-C^{5}\right)=4.5 \mathrm{~Hz}\right) ; 110.55\left(d, C-6,4 J\left(F-C^{6}\right)=3.4 \mathrm{~Hz}\right)$; $103.86\left(\mathrm{~d}, \mathrm{C}-2, J\left(\mathrm{~F}-\mathrm{C}^{2}\right)=23.9 \mathrm{~Hz}\right) ; 52.97(\mathrm{~d}, \mathrm{C}-7$ and $\mathrm{C}-11$, $\left.4 J\left(\mathrm{~F}-\mathrm{C}^{7(11)}\right)=2.5 \mathrm{~Hz}\right) ; 26.31(\mathrm{C}-8, \mathrm{C}-10) ; 24.18(\mathrm{C}-9)$.

\section{Synthesis of 3-fluoro-4-mopholinyl-aniline}

3-fluoro-4-morfolinyl-aniline: (m.p. $120.2-122.2^{\circ} \mathrm{C}$, yield 95\%). ${ }^{1} \mathrm{H}-\mathrm{NMR}\left(\mathrm{CDCl}_{3^{\prime}} \delta \mathrm{ppm}, j \mathrm{~Hz}\right): 6.79(\mathrm{t}, 1 \mathrm{H}, \mathrm{H}-5,4 J(\mathrm{~F}-$ $\left.\left.\mathrm{H}^{5}\right)=3\left(\mathrm{H}^{5}-\mathrm{H}^{6}\right)=9.2\right) ; 6.46 \div 6.38(\mathrm{~m}, 2 \mathrm{H}, \mathrm{H}-2, \mathrm{H}-6) ; 3.84(\mathrm{~m}$, $4 \mathrm{H}, \mathrm{H}-8, \mathrm{H}-10$, syst. $\left.\mathrm{A}_{2} \mathrm{~B}_{2}\right) ; 2.95\left(\mathrm{~m}, 4 \mathrm{H}, \mathrm{H}-7, \mathrm{H}-11\right.$, syst. $\mathrm{A}_{2} \mathrm{~B}_{2}$ ). ${ }^{13} \mathrm{C}-\mathrm{NMR}\left(\mathrm{CDCl}{ }_{3,} \delta \mathrm{ppm}\right): 156.70\left(\mathrm{~d}, \mathrm{C}-3, J\left(\mathrm{~F}-\mathrm{C}^{3}\right)=244.8\right.$ $\mathrm{Hz}) ; 143.20\left(\mathrm{~d}, \mathrm{C}-1,3\left(\mathrm{~F}-\mathrm{C}^{1}\right)=10.6 \mathrm{~Hz}\right) ; 131.32(\mathrm{~d}, \mathrm{C}-4, J(\mathrm{~F}-$ $\left.\left.\mathrm{C}^{4}\right)=10.0 \mathrm{~Hz}\right) ; 120.18\left(\mathrm{~d}, \mathrm{C}-5,3 \mathrm{3}\left(\mathrm{F}-\mathrm{C}^{5}\right)=4.6 \mathrm{~Hz}\right) ; 110.56(\mathrm{~d}$, $\left.\mathrm{C}-6,4 J\left(\mathrm{~F}-\mathrm{C}^{6}\right)=2.8 \mathrm{~Hz}\right) ; 103.73\left(\mathrm{~d}, \mathrm{C}-2, J\left(\mathrm{~F}-\mathrm{C}^{2}\right)=23.5\right.$ $\mathrm{Hz}) ; 67.08(\mathrm{C}-8, \mathrm{C}-10) ; 51.72\left(\mathrm{~d}, \mathrm{C}-7, \mathrm{C}-11, J\left(\mathrm{~F}-\mathrm{C}^{7,11}\right)=2.0 \mathrm{~Hz}\right)$.
Synthesis of N-Benzyloxycarbonyl-3-fluoro-4-(3-methylpiperidinyl)-aniline

To a mixture of $0.027 \mathrm{~mol}$ (5.32 g) 3-fluoro-4-(3-methylpiperidinyl)-aniline, $0.065 \mathrm{~mol}(5.4 \mathrm{~g})$ sodium bicarbonate in $100 \mathrm{~mL}$ acetone and $50 \mathrm{~mL}$ water cooled at $5^{\circ} \mathrm{C}$ was added drop wise for $20-30 \mathrm{~min}$., $0.033 \mathrm{~mol}(5.63 \mathrm{~g})$ benzyl chloroformate, while maintaining the temperature between 5 and $10^{\circ} \mathrm{C}$, and then allowed to stir at room temperature $4 \mathrm{~h}$. The mixture was poured over $500 \mathrm{~g}$ ice and $100 \mathrm{~mL}$ of water. The mixture was filtered, and the solids were washed with water, and then dried to give 8.13 g N-Benzyloxycarbonyl-3-fluoro-4-(3-methyl-piperidinyl)aniline (109.3-111.60 $\mathrm{C}$; yield 88\%). ${ }^{\mathrm{H}} \mathrm{H}-\mathrm{NMR}\left(\mathrm{CDCl}_{3}, \delta \mathrm{ppm}\right.$, J Hz): $7.41 \div 7.33\left(\mathrm{~m}, 6 \mathrm{H}, \mathrm{H}-2, \mathrm{H}^{15} \div \mathrm{H}^{19}\right) ; 6.94\left(\mathrm{dd},{ }^{3}{ }^{\prime} \mathrm{H}, \mathrm{H}-6\right.$, $\left.2.2,8.8) ; 6.88\left(\mathrm{t}, 1 \mathrm{H}, \mathrm{H}-5,{ }^{4}\right)\left(\mathrm{F}-\mathrm{H}^{5}\right)=3\left(\mathrm{H}^{5}-\mathrm{H}^{6}\right)=8.8\right) ; 6.58(\mathrm{bs}$, $\mathrm{NH}) ; 3.27\left(\mathrm{~m}, 2 \mathrm{H}, \mathrm{H}-7, \mathrm{H}-11_{\text {or }} \mathrm{H}-7, \mathrm{H}-11\right) ; 2.53(\mathrm{~m}$, $1 \mathrm{H}, \mathrm{H}-8) ; 5.18\left(\mathrm{~s}, 2 \mathrm{H}, \mathrm{H}^{\mathrm{a}} \mathrm{I}^{\prime} 3\right) ; 2.23\left(\mathrm{~m}, 2 \mathrm{H}^{\mathrm{q}}, \mathrm{H}-7 \mathrm{e}, \mathrm{eq}-11\right.$ or $\mathrm{H}^{\prime}$ 7 , H-11 $) ; 1.85 \div 1.72(\mathrm{~m}, 4 \mathrm{H}, \mathrm{H}-9, \mathrm{H}-10) ; 0.92\left(\mathrm{~d}, \mathrm{eq}^{\mathrm{H}}, \mathrm{H}-\right.$ $\left.20 x^{2}, 6.4\right){ }^{a x}{ }_{13} \mathrm{C}-\mathrm{NMR}\left(\mathrm{CDCl}{ }_{3}, \delta \mathrm{ppm}\right): 155.72(\mathrm{~d}, \mathrm{C}-3, J(\mathrm{~F}-$ $\left.\left.\mathrm{C}^{3}\right)=245.4 \mathrm{~Hz}\right) ; 153.37\left(\mathrm{C}^{3}-12\right) ; 137.40(\mathrm{~d}, \mathrm{C}-1,3)\left(\mathrm{F}-\mathrm{C}^{1}\right)=9.7$ $\mathrm{Hz}) ; 132.36\left(\mathrm{~d}, \mathrm{C}-4, J\left(\mathrm{~F}-\mathrm{C}^{4}\right)=10.2 \mathrm{~Hz}\right) ; 135.97(\mathrm{C}-14)$; 128.63(C-16, C-18); 28.34(C-17); 128.33(C-15, C19); $\left.119.50(\mathrm{~d}, \mathrm{C}-5,3)\left(\mathrm{F}-\mathrm{C}^{5}\right)=4.4 \mathrm{~Hz}\right) ; 114.38(\mathrm{~d}, \mathrm{C}-6,4)(\mathrm{F}-$ $\left.\left.\mathrm{C}^{6}\right)=3.4 \mathrm{~Hz}\right) ; 107.58\left(\mathrm{~d}, \mathrm{C}-2, \mathrm{~J}\left(\mathrm{~F}-\mathrm{C}^{2}\right)=22.7 \mathrm{~Hz}\right) ; 67.06(\mathrm{C}-12)$; $59.43(\mathrm{C}-7$ or $\mathrm{C}-11) ; 51.79(\mathrm{C}-7$ or $\mathrm{C}-11) ; 32.76(\mathrm{C}-9$ or $\mathrm{C}-$ 10); 31.29(C-9 or C-10); 25.68(C-8); 19.89(C-20). Elemental Analyses: Calculated for: $\mathrm{C}_{22} \mathrm{H}_{23} \mathrm{FN} \mathrm{O}_{2}: \mathrm{C}: 70.15 \% \mathrm{H}: 6.77 \%$ $\mathrm{N}: 8.18 \%$. Found $\mathrm{C}: 70.22 \%{ }^{2} \mathrm{H}: \mathrm{b}^{2 \mathrm{6}} .92 \%{ }^{2} \mathrm{~N}: 8.22 \%$.

Synthesis of N-Benzyloxycarbonyl-3-fluoro-4-(4-methylpiperidinyl)-aniline

N-Benzyloxycarbonyl-3-fluoro-4-(4-methyl-piperidinyl)aniline: (m.p.121,5-123,40 ; yield $97 \%$ ). ${ }^{1} \mathrm{H}-\mathrm{NMR}\left(\mathrm{CDCl}_{3}, \delta\right.$ ppm, J Hz): 7.42 $\div 7.34\left(\mathrm{~m}, 6 \mathrm{H}, \mathrm{H}-2, \mathrm{H}^{15} \div \mathrm{H}^{19}\right) ; 6.94\left(\mathrm{dd}, \mathrm{1}^{\prime} \mathrm{H}\right.$, $H-6,2.2,8.8) ; 6.88\left(t, 1 H, H-5,4 J\left(F-H^{5}\right)=3\left(H^{5}-H^{6}\right)=8.8\right)$; 6.61(bs $1 \mathrm{H}, \mathrm{HN}) ; 5.18(\mathrm{~s}, 2 \mathrm{H}, \mathrm{H}-13) ; 3.33(\mathrm{~m}, 2 \mathrm{H}, \mathrm{H}-7$, H$11_{\text {eq }}$ or $\left.\mathrm{H}-7_{\mathrm{ax}} \mathrm{H}^{\prime}-11_{\mathrm{ax}}\right) ; 2.60\left(\mathrm{~m}, 2 \mathrm{H}, \mathrm{H}-\mathrm{f}_{\mathrm{eq}} \mathrm{H}-11_{\text {of }}\right.$ or $\mathrm{H}-\mathrm{7}_{\mathrm{ax}}^{\mathrm{eq}} \mathrm{H}-$

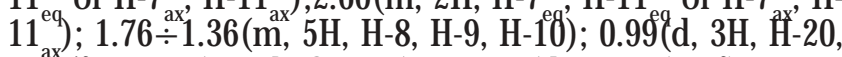
5.5). ${ }^{33} \mathrm{C}-\mathrm{NMR}\left(\mathrm{CDCl}{ }_{3}, \delta \mathrm{ppm}\right): 156.02\left(\mathrm{~d}, \mathrm{C}-3, J\left(\mathrm{~F}-\mathrm{C}^{3}\right)=244.7\right.$ $\mathrm{Hz}) ; \quad 154.06(\mathrm{C}-12) ; 137.35(\mathrm{~d}, C-1,3)\left(\mathrm{F}-\mathrm{C}^{1}\right)=10.1$ $\mathrm{Hz}) ; 132.31\left(\mathrm{~d}, \mathrm{C}-4, J\left(\mathrm{~F}^{-} \mathrm{C}^{4}\right)=10.4 \mathrm{~Hz}\right) ; 135.97(\mathrm{C}-14)$; 128.62 (C-16, C-18); 128.38 (C-17); 128.33 (C-15, C19); $\left.119.43(\mathrm{~d}, \mathrm{C}-5,3)\left(\mathrm{F}-\mathrm{C}^{5}\right)=4.4 \mathrm{~Hz}\right) ; 114.33(\mathrm{~d}, \mathrm{C}-6,4)(\mathrm{F}-$ $\left.\left.\mathrm{C}^{6}\right)=3.4 \mathrm{~Hz}\right) ; 107.59\left(\mathrm{~d}, \mathrm{C}-2, J\left(\mathrm{~F}-\mathrm{C}^{2}\right)=22.7 \mathrm{~Hz}\right) ; 67.06(\mathrm{C}-13)$; 51.69(C-7, C-11); 34.44(C-8, C-10);30.58(C-9); 21.94(C-20). Elemental Analyses: Calculated for: $\mathrm{C}_{20} \mathrm{H}_{22} \mathrm{FN} \mathrm{O}_{2}: \mathrm{C}: 70.15 \%$ $\mathrm{H}: 6.77 \% \mathrm{~N}: 8.18 \%$. Found C: $70.17 \% \mathrm{H}:{ }^{2} 6.70 \% \mathrm{~N}: 8.33 \%$

Synthesis of N-Benzyloxycarbonyl-3-fluoro-4-piperidinylaniline

N-Benzyloxycarbonyl-3-fluoro-4-piperidinyl-aniline: (m.p. $116.4-118.5^{\circ} \mathrm{C}$; yield $68.2 \%$ ). ${ }^{1} \mathrm{H}-\mathrm{NMR}\left(\mathrm{CDCl}_{3}, \delta \mathrm{ppm}\right.$, J Hz): $7.40 \div 7.32\left(\mathrm{~m}, 6 \mathrm{H}, \mathrm{H}-2, \mathrm{H}^{15} \div \mathrm{H}^{19}\right) ; 6.95\left(\mathrm{dd}^{3^{\prime}}, 1 \mathrm{H}, \mathrm{H}-6\right.$, $2.1,8.8) ; 6.87\left(\mathrm{t}, 1 \mathrm{H}, \mathrm{H}-5,{ }^{4} J\left(\mathrm{~F}-\mathrm{H}^{5}\right)=3\left(\mathrm{H}^{5}-\mathrm{H}^{6}\right)=8.8\right) ; 6.63(\mathrm{bS}$, $1 \mathrm{H}, \mathrm{NH}) ; 5.18(\mathrm{~s}, 2 \mathrm{H}, \mathrm{H}-13) ; 2.95(\mathrm{t}, 4 \mathrm{H}, \mathrm{H}-7, \mathrm{H}-11,5.4)$; $1.72(\mathrm{qv}, 4 \mathrm{H}, \mathrm{H}-8, \mathrm{H}-10,5.4) ; 1.56(\mathrm{~m}, 2 \mathrm{H}, \mathrm{H}-9) .{ }^{13} \mathrm{C}-$ $\operatorname{NMR}\left(\mathrm{CDCl}_{3}, \delta \mathrm{ppm}\right): 155.70\left(\mathrm{~d}, \mathrm{C}-3, J\left(\mathrm{~F}-\mathrm{C}^{3}\right)=244.6 \mathrm{~Hz}\right)$; 153.33(C-12); 137.42(d, C-1, 3J(F-C 1$)=9.3 \mathrm{~Hz}) ; 132.34(\mathrm{~d}, \mathrm{C}-$ $4, J\left(F-C^{4}\right)=10.8 \mathrm{~Hz}$ );135.97(C-14); 128.62 (C-16, C-18); 128.37(C-17); 128.32(C-15, C-19);119.39(d, C-5, 3J(F$\left.\left.\left.\mathrm{C}^{5}\right)=4.4 \mathrm{~Hz}\right) ; 114.41(\mathrm{~d}, \mathrm{C}-6,4)\left(\mathrm{F}-\mathrm{C}^{6}\right)=3.4 \mathrm{~Hz}\right) ; 107.55(\mathrm{~d}, \mathrm{C}-$ $\left.2, J\left(F-C^{2}\right)=22.7 \mathrm{~Hz}\right) ; 67.05(\mathrm{C}-12) ; 52.34(\mathrm{C}-7, \mathrm{C}-11)$; 26.16(C08, C-10); 14.17(C-9). Elemental Analyses: Calculated for: $\mathrm{C}_{10} \mathrm{H}_{22} \mathrm{FN}_{2} \mathrm{O}_{2}$ : C: $69.49 \% \mathrm{H:}: 6.45 \% \mathrm{~N}: 8.53 \%$. Found C: $70.17 \%{ }^{19} \mathrm{H}:{ }^{21} .70 \% \% \mathrm{~N}: 8.33 \%$

Synthesis of N-Benzyloxycarbonyl-3-fluoro-4-morpholinyl-aniline

N-Benzyloxycarbonyl-3-fluoro-4-mofolinyl-aniline: (m.p. $123-123.7^{\circ} \mathrm{C}$; yield 96.6\%). ${ }^{1} \mathrm{H}-\mathrm{NMR}\left(\mathrm{CDCl}_{3}, \delta \mathrm{ppm}, \mathrm{J}_{\mathrm{Hz}}\right.$ : $7.40 \div 7.32\left(\mathrm{~m}, 6 \mathrm{H}, \mathrm{H}-2, \mathrm{H}^{15} \div \mathrm{H}^{19}\right) ; 6.95(\mathrm{dd}, 1 \mathrm{H}, \mathrm{H}-6,2.1,8.8)$; 
$6.87\left(\mathrm{t}, \mathrm{H}, \mathrm{H}-5,{ }^{4}\left(\mathrm{~F}-\mathrm{H}^{5}\right)={ }^{3}\left(\mathrm{H}^{5}-\mathrm{H}^{6}\right)=8.8\right) ; 6.63(\mathrm{bs}, 1 \mathrm{H}, \mathrm{NH})$; 5.18(s, 2H, H-13); 2.95(t, 4H, H-7, H-11, 5.4); $1.72(\mathrm{qv}, 4 \mathrm{H}$, $\mathrm{H}-8, \mathrm{H}-10,5.4) ; 1.56(\mathrm{~m}, 2 \mathrm{H}, \mathrm{H}-9) .{ }^{13} \mathrm{C}-\mathrm{NMR}\left(\mathrm{CDCl}{ }_{3}, \delta \mathrm{ppm}\right)$ : $155.70\left(\mathrm{~d}, \mathrm{C}-3, J\left(\mathrm{~F}-\mathrm{C}^{3}\right)=244.6 \mathrm{~Hz}\right) ; 153.33(\mathrm{C}-12) ; 137.42(\mathrm{~d}$, $\left.\mathrm{C}-1,3\left(\mathrm{~F}-\mathrm{C}^{1}\right)=9.3 \mathrm{~Hz}\right) ; 132.34\left(\mathrm{~d}, \mathrm{C}-4, J\left(\mathrm{~F}-\mathrm{C}^{4}\right)=10.8\right.$ $\mathrm{Hz}) ; 135.97(\mathrm{C}-14) ; 128.62(\mathrm{C}-16, \mathrm{C}-18) ; 128.37(\mathrm{C}-17)$; 128.32(C-15, C-19);119.39(d, C-5, 3/ (F-C $\left.\left.{ }^{5}\right)=4.4 \mathrm{~Hz}\right)$; $\left.114.41(\mathrm{~d}, \mathrm{C}-6,4)\left(\mathrm{F}-\mathrm{C}^{6}\right)=3.4 \mathrm{~Hz}\right) ; 107.55\left(\mathrm{~d}, \mathrm{C}-2, J\left(\mathrm{~F}-\mathrm{C}^{2}\right)=22.7\right.$ $\mathrm{Hz}) ; 67.05(\mathrm{C}-12) ; 52.34(\mathrm{C}-7, \mathrm{C}-11) ; 26.16(\mathrm{C} 08, \mathrm{C}-10)$; 14.17(C-9). Elemental Analyses: Calculated for: $\mathrm{C}_{1} \mathrm{H}_{1} \mathrm{FN}_{2}$ : C: $65.44 \% \mathrm{H}: 5.80 \% \mathrm{~N}: 8.48 \%$. Found C: C : $65.51 \%$ H: $5.90 \%$ N:8.51\%

Synthesis of (R)-[N-3-(3-fluoro-4-(3-methyl-piperidinylphenyl)-2-oxo-5-oxazolidinyl]methanol (Alcohol 4)

To a mixture of $0.0207 \mathrm{~mol}(7.08 \mathrm{~g})$ of N-Benzyloxycarbonyl-3-fluoro-4-(3-methyl-piperidinyl)-aniline in $100 \mathrm{~mL}$ tetrahydrofuran anh. at $-78^{\circ} \mathrm{C}$, under argon was added $0.207 \mathrm{~mol}(8.4 \mathrm{~mL}) 2.5 \mathrm{M} \mathrm{nBuLi}$ - hexane drop wise over 20-30 min. After $35 \mathrm{~min} .0 .021 \mathrm{~mol}(3.05 \mathrm{~g})$ of (R)-glycidyl butyrate was added and the mixture allowed to stir at $-78^{\circ}$ $\mathrm{C}$ for $40 \mathrm{~min}$. and then at room temperature for $24 \mathrm{~h}$. Saturated aqueous ammonium chloride ( $30 \mathrm{~mL}$ ) was added followed by $60 \mathrm{~mL}$ ethyl acetate and $20 \mathrm{~mL}$ water. The layers were separated, and the aqueous layer was extracted with ethyl acetate $(3 \times 30 \mathrm{~mL})$. The combined organic layers were dried $\left(\mathrm{Na}_{3} \mathrm{SO}_{4}\right)$ and concentrated under reduced pressure to give after recrystallized from ethyl acetate-hexane $5.0 \mathrm{~g}(R)$-[N-3-(3-fluoro-4-(3-methylpiperidinyl-phenyl)-2-oxo-5-oxazolidinyl]methanol (m.p. 98.6-101.30 $3^{\circ}$, yield 78\%). ${ }^{1} \mathrm{H}-\mathrm{NMR}(\mathrm{CDCl}{ }, \delta \mathrm{ppm}, J \mathrm{~Hz})$ : $\left.\left.7.38\left(\mathrm{dd}, 1 \mathrm{H}, \mathrm{H}-2,{ }^{4}\right)\left(\mathrm{H}^{6}-\mathrm{H}^{2}\right)=2.1,4\right)\left(\mathrm{F}-\mathrm{H}^{2}\right)=14.4\right) ; 7.10(\mathrm{dd}$, $\left.1 \mathrm{H}, \mathrm{H}-6,5)\left(\mathrm{F}-\mathrm{H}^{6}\right)=2.1,4 J\left(\mathrm{H}^{5}-\mathrm{H}^{6}\right)=9.1\right) ; 6.93\left(\mathrm{t}, 1 \mathrm{H}, \mathrm{H}-5,{ }^{3}\right)\left(\mathrm{H}^{5}-\right.$ $\left.\left.H^{6}\right)=9.1, J\left(F-H^{5}\right)=9.1\right) ; 4.72(m, 1 H, H-13) ; 4.00(d, 1 H, H-$ $15 \mathrm{~A}, 8.7) ; 3.94(\mathrm{dd}, 1 \mathrm{H}, \mathrm{H}-15 \mathrm{~B}, 8.7,6.8) ; 3.96(\mathrm{~d}, 1 \mathrm{H}, \mathrm{H}-14 \mathrm{~A}$, 12.6); $3.74(\mathrm{dd}, 1 \mathrm{H}, \mathrm{H}-14 \mathrm{~B}, 12.6,4.2) ; 3.30(\mathrm{~m}, 2 \mathrm{H}, \mathrm{H}-11)$; 2.70 (bs, $1 \mathrm{H}, \mathrm{OH}) ; 2.55(\mathrm{~m}, 1 \mathrm{H}, \mathrm{H}-7) ; 2.26(\mathrm{~m}, \mathrm{H}-7) ; 1.97(\mathrm{~m}$, $5 \mathrm{H}, \mathrm{H}-8,2 \mathrm{H}-9,2 \mathrm{H}-10) ; 0.93\left(\mathrm{~d}, 3 \mathrm{H}, \mathrm{H}-\mathrm{8}^{\prime}, 5.9\right) .{ }^{13} \mathrm{C}-\mathrm{NMR}(\mathrm{CDCl}{ }$ $\delta \mathrm{ppm}): 155.46\left(\mathrm{~d}, \mathrm{C}-3, J\left(\mathrm{~F}-\mathrm{C}^{3}\right)=243.2 \mathrm{~Hz}\right) ; 154.64\left(\mathrm{C}^{-}\right.$ 12); $137.81\left(\mathrm{~d}, \mathrm{C}-4, J\left(\mathrm{~F}-\mathrm{C}^{4}\right)=9.3 \mathrm{~Hz}\right) ; 132.89(\mathrm{~d}, \mathrm{C}-1, J(\mathrm{~F}-$ $\left.\left.\mathrm{C}^{4}\right)=10.3 \mathrm{~Hz}\right) ; 119.25\left(\mathrm{~d}, \mathrm{C}-5, J\left(\mathrm{~F}-\mathrm{C}^{5}\right)=3.8 \mathrm{~Hz}\right) ; 113.87(\mathrm{~d}, \mathrm{C}-$ 6 , $\left.J\left(F-C^{6}\right)=3.7 \mathrm{~Hz}\right) ; 107.32\left(\mathrm{~d}, \mathrm{C}-2, J\left(\mathrm{~F}-\mathrm{C}^{2}\right)=26.3 \mathrm{~Hz}\right)$; 72.83(C-13); 62.75(C-15);46.49(C-14);59.28(C-11); $51.65\left(\mathrm{~d}, \mathrm{C}-7, \mathrm{C}-11, J\left(\mathrm{~F}-\mathrm{C}^{7,11}\right)=3.2 \mathrm{~Hz}\right) ; 32.77(\mathrm{C}-10) ; 31.26(\mathrm{C}-$ 8); $25.64\left(\right.$ C-9); $19.48\left(C-8^{\prime}\right)$. Elemental Analyses: Calculated for $\mathrm{C}_{1} \mathrm{H}_{21} \mathrm{FN}_{2} \mathrm{O}_{3}$ : C: $62.32 \% \mathrm{H}: 6.86 \% \mathrm{~N}: 9.09 \%$. Found $\mathrm{C}: 62.19 \% \mathrm{H}^{21}: 7.00 \% \mathrm{~N}: 9.27 \%$

Synthesis of (R)-[N-3-(3-fluoro-4-(4-methyl-piperidinylphenyl)-2-oxo-5-oxazolidinyl]methanol (Alcohol 5)

(R)-[N-3-(3-fluoro-4-(3-methyl-piperidinyl-phenyl)-2oxo-5-oxazolidinyl]methanol (m.p. $121.5-123.4^{\circ} \mathrm{C}$, yield 75\%). ${ }^{1} \mathrm{H}-\mathrm{NMR}\left(\mathrm{CDCl}_{3}, \delta \mathrm{ppm}, J, \mathrm{~Hz}\right): 7.38\left(\mathrm{dd}, 1 \mathrm{H}, \mathrm{H}-2,{ }^{4}{ }^{4}\left(\mathrm{H}^{6}-\right.\right.$ $\left.\left.\mathrm{H}^{2}\right)=1.9,4 J\left(\mathrm{~F}-\mathrm{H}^{2}\right)=14.4\right) ; 7.08(\mathrm{dd}, 1 \mathrm{H}, \mathrm{H}-6,5)\left(\mathrm{F}-\mathrm{H}^{6}\right)=1.9$, $\left.4 J\left(H^{5}-H^{6}\right)=9.1\right) ; 6.93\left(t, \quad 1 H, H-5,3\left(H^{5}-H^{6}\right)=8.7\right.$, J(F$\left.\left.\mathrm{H}^{5}\right)=9.1\right) ; 4.70(\mathrm{~m}, 1 \mathrm{H}, \mathrm{H}-13) ; 3.99(\mathrm{~m}, 1 \mathrm{H}, \mathrm{H}-15 \mathrm{~A}, J(\mathrm{~A}-$ $\left.B)=16.9, J\left(H^{15 A}-H^{13}\right)=8.7\right) ; 3.93(m, 1 H, H-15 B, J(A-$ $B)=16.9, J\left(\mathrm{H}^{15 B}-\mathrm{H}^{13}=8.7\right) ; 3.92(\mathrm{~m}, 1 \mathrm{H}, \mathrm{H}-14 \mathrm{~A}) ; 3.72(\mathrm{dd}, 1 \mathrm{H}$, $\left.\mathrm{H}-14 \mathrm{~B}, J(\mathrm{~A}-\mathrm{B})=12.6, J\left(\mathrm{H}^{13}-\mathrm{H}^{14 \mathrm{~B}}\right)=3.9\right) ; 3.35(\mathrm{~m}, 2 \mathrm{H}, \mathrm{H}-7 \mathrm{eq}$, $\mathrm{H}$-1leq or H-7ax, H-1lax); 3.13(bs, $1 \mathrm{H}, \mathrm{OH}) ; 2.62(\mathrm{~m}, 2 \mathrm{H}$, $\mathrm{H}-7 e q, \mathrm{H}-1$ leq or H-7ax, H-11ax); $1.73(\mathrm{~m}, 2 \mathrm{H}, \mathrm{H}-8 \mathrm{eq}, \mathrm{H}-$ 10eq or H-8ax, H-10ax); $1.32 \div 1.48(\mathrm{~m}, 3 \mathrm{H}, \mathrm{H}-13, \mathrm{H}-8 \mathrm{eq}$, $\mathrm{H}-10 \mathrm{eq}$ or H-8ax, $\mathrm{H}-10 \mathrm{ax}) ; 0.98\left(\mathrm{~d}, 3 \mathrm{H}, \mathrm{H}-9^{\prime}, 5.8\right)$. $4.01 \div 3.89(\mathrm{~m}, 3 \mathrm{H}, \mathrm{H}-14 \mathrm{~A}, \mathrm{H}-15 \mathrm{~A}, \mathrm{H}-15 \mathrm{~B}) \cdot{ }^{13} \mathrm{C}-\mathrm{NMR}(\mathrm{CDCl}$, $\delta \mathrm{ppm}): 155.42\left(\mathrm{~d}, \mathrm{C}-3, J\left(\mathrm{~F}-\mathrm{C}^{3}\right)=245.1 \mathrm{~Hz}\right) ; 154.73\left(\mathrm{C}^{\prime}\right.$ 12); $137.56\left(\mathrm{~d}, \mathrm{C}-4, J\left(\mathrm{~F}-\mathrm{C}^{4}\right)=8.8 \mathrm{~Hz}\right) ; 132.46(\mathrm{~d}, \mathrm{C}-1, J(\mathrm{~F}-$ $\left.\left.C^{4}\right)=10.3 \mathrm{~Hz}\right) ; 119.25\left(\mathrm{~d}, \mathrm{C}-5, J\left(\mathrm{~F}-\mathrm{C}^{5}\right)=4.5 \mathrm{~Hz}\right) ; 113.86(\mathrm{~d}, \mathrm{C}-$ $\left.6, J\left(F-C^{6}\right)=3.3 \mathrm{~Hz}\right) ; 107.31\left(\mathrm{~d}, \mathrm{C}-2, J\left(\mathrm{~F}^{\left.-\mathrm{C}^{2}\right)}=26.1 \mathrm{~Hz}\right)\right.$; 72.92(C-13); 62.66(C-15); 51.57(C-7, C-11); 46.51(C-14); $34.41(C-8, C-10) ; 30.59(C-9) ; 21.93\left(C-9^{\prime}\right)$. Elemental
Analyses: Calculated for $\mathrm{C}_{1} \mathrm{H}_{2} \mathrm{FN}_{\mathrm{O}}$ : $\mathrm{C}: 62.32 \% \mathrm{H:}: 6.86 \%$ $\mathrm{N}: 9.09 \%$. Found $\mathrm{C}: 62.37 \% \mathrm{H}^{16} \mathrm{H}: 6.80 \% \mathrm{~N}: 9.23 \%$.

Synthesis of (R)-[N-3-(3-fluoro-4-(piperidinyl-phenyl)2-oxo-5-oxazolidinyl]methanol (Alchool 3)

$(R)$-[N-3-(3-fluoro-4-(piperidinyl-phenyl)-2-0x0-5oxazolidinyl]methanol: (m.p. $116.4-118.5^{\circ} \mathrm{C}$, yield $77 \%$ ).

p.t.116,4-118,50 ${ }^{1}{ }^{1} \mathrm{H}-\mathrm{NMR}\left(\mathrm{CDCl}_{3}, \delta \mathrm{ppm}, J \mathrm{~Hz}\right): 7.38(\mathrm{dd}$, $\left.1 \mathrm{H}, \mathrm{H}-2,4 J\left(\mathrm{~F}-\mathrm{H}^{2}\right)=2.1,4 J\left(\mathrm{H}^{6}-\mathrm{H}^{2}\right)=14.4\right) ; 7.10(\mathrm{dd}, 1 \mathrm{H}, \mathrm{H}-6$, $\left.5 /\left(F-H^{6}\right)=2.1,4 J\left(H^{5}-H^{6}\right)=9.1\right) ; 6.93\left(t, 1 H, H-5,3\left(H^{5}-H^{6}\right)=9.1\right.$, $\left.J\left(F-H^{5}\right)=9.1\right) ; 4.72(m, 1 H, H-13) ; 4.00(d, 1 H, H-15 A, 8.7)$; 3.92(dd, $1 \mathrm{H}, \mathrm{H}-15 \mathrm{~B}, 8.7,6.8) ; 3.96(\mathrm{~d}, 1 \mathrm{H}, \mathrm{H}-14 \mathrm{~A}, 12.6)$; 3.74(dd, $1 \mathrm{H}, \mathrm{H}-14 \mathrm{~B}, 12.6,4.2) ; 2.98(\mathrm{~m}, 4 \mathrm{H}, \mathrm{H}-7, \mathrm{H}-11)$; 2.70(bs, $1 \mathrm{H}, \mathrm{OH}$ ); 1.70 (qv, 4H, H-8, H-10, 5.6); $1.58(\mathrm{~m}, 2 \mathrm{H}$, H-9). ${ }^{13} \mathrm{C}-\mathrm{NMR}\left(\mathrm{CDCl}_{3}, \delta \mathrm{ppm}\right): 155.46\left(\mathrm{~d}, \mathrm{C}-3, \mathrm{~J}\left(\mathrm{~F}-\mathrm{C}^{3}\right)=243.2\right.$ $\mathrm{Hz}) ; 154.64(\mathrm{C}-12) ; 137.81\left(\mathrm{~d}, \mathrm{C}-4, J\left(\mathrm{~F}-\mathrm{C}^{4}\right)=9.3 \mathrm{~Hz}\right) ; 132.89(\mathrm{~d}$, $\left.\mathrm{C}-1, J\left(\mathrm{~F}-\mathrm{C}^{4}\right)=10.3 \mathrm{~Hz}\right) ; 119.22\left(\mathrm{~d}, \mathrm{C}-5, J\left(\mathrm{~F}-\mathrm{C}^{5}\right)=3.8 \mathrm{~Hz}\right)$; $113.86\left(\mathrm{~d}, \mathrm{C}-6, J\left(\mathrm{~F}-\mathrm{C}^{6}\right)=3.7 \mathrm{~Hz}\right) ; 107.32\left(\mathrm{~d}, \mathrm{C}-2, J\left(\mathrm{~F}-\mathrm{C}^{2}\right)=26.3\right.$ $\mathrm{Hz}) ; 72.83(\mathrm{C}-13) ; 62.75(\mathrm{C}-15) ; 52.22(\mathrm{~d}, \mathrm{C}-7, \mathrm{C}-11, J(\mathrm{~F}-$ $\left.\left.\mathrm{C}^{7,11}\right)=3.2 \mathrm{~Hz}\right) ; 46.48(\mathrm{C}-14) ; 26.14(\mathrm{C}-8, \mathrm{C}-10) ; 24.19(\mathrm{C}$ 9).Elemental Analyses: Calculated for $\mathrm{C}_{15} \mathrm{H}_{1 \mathrm{FN}} \mathrm{FN}_{2}$ : $\mathrm{C}$ : $61.21 \% \mathrm{H}: 6.51 \% \mathrm{~N}: 9.52 \%$. Found: C : $61.02 \% \mathrm{H}: 6.70 \% \mathrm{~N}$ : $9.50 \%$

Synthesis of (R)-[N-3-(3-fluoro-4-(morpholinyl-fenil)-2oxo-5-oxazolidinyl]methanol (Alcohol 1)

(R)-[N-3-(3-fluoro-4-(morpholinyl-phenyl)-2-0xo-5oxazolidinyl]methanol: (m.p. $111.7-112.5^{\circ} \mathrm{C}$, yield $75 \%$ ). ${ }^{1} \mathrm{H}$ $\mathrm{NMR}\left(\mathrm{CDCl}_{3}, \delta \mathrm{ppm}, J \mathrm{~Hz}\right): 7.43\left(\mathrm{dd}, 1 \mathrm{H}, \mathrm{H}-2,4 J\left(\mathrm{~F}-\mathrm{H}^{2}\right)=1.9\right.$, $\left.{ }^{4}\left(\mathrm{H}^{6}-\mathrm{H}^{2}\right)=14.4\right) ; 7.10(\mathrm{dd}, 1 \mathrm{H}, \mathrm{H}-6,5)\left(\mathrm{F}-\mathrm{H}^{6}\right)=1.9,4{ }^{4}\left(\mathrm{H}^{5}-\right.$ $\left.\left.\mathrm{H}^{6}\right)=9.1\right) ; 6.91\left(\mathrm{t}, 1 \mathrm{H}, \mathrm{H}-5,{ }^{3}\left(\mathrm{H}^{5}-\mathrm{H}^{6}\right)=8.7, J\left(\mathrm{~F}-\mathrm{H}^{5}\right)=8.7\right)$; $3.86\left(\mathrm{~m}, 4 \mathrm{H}, \mathrm{H}-8, \mathrm{H}-10\right.$, syst. $\left.\mathrm{A}_{2} \mathrm{~B}_{2}\right) ; 3.14(\mathrm{bs}, 1 \mathrm{H}, \mathrm{HO})$; 3.04( $\mathrm{m}, 4 \mathrm{H}, \mathrm{H}-7, \mathrm{H}-11$, syst. $\left.\mathrm{A}{ }_{2} \mathrm{~B}^{2}\right) ; 4.73(\mathrm{~m}, 1 \mathrm{H}, \mathrm{H}-13)$; 3.99(d, $1 \mathrm{H}, H-15 \mathrm{~A}$, syst. $A B, 8.6) ; 3.94(\mathrm{dd}, 1 \mathrm{H}, \mathrm{H}-15 \mathrm{~B}$, syst $\left.A B, 8.6, J\left(H^{15 B}-H^{13}\right)=6.8 \mathrm{~Hz}\right) ; 3.97(\mathrm{bd}, 1 \mathrm{H} . \mathrm{H}-14 \mathrm{~A}, 11.4)$; 3.73(bd, $1 \mathrm{H}, \mathrm{H}-14 \mathrm{~B}$, syst. AB, 11.4). ${ }^{13} \mathrm{C}-\mathrm{NMR}(\mathrm{CDCl}{ }, \delta \mathrm{ppm})$ : $155.34\left(\mathrm{~d}, \mathrm{C}-3, J\left(\mathrm{~F}-\mathrm{C}^{3}\right)=244.4 \mathrm{~Hz}\right) ; 154.71(\mathrm{C}-12) ; 136.25(\mathrm{~d}$, $\left.\mathrm{C}-4, J\left(\mathrm{~F}-\mathrm{C}^{4}\right)=9.1 \mathrm{~Hz}\right) ; 133.11\left(\mathrm{~d}, \mathrm{C}-1, J\left(\mathrm{~F}-\mathrm{C}^{4}\right)=10.5 \mathrm{~Hz}\right)$; 118.73(d, C-5, J(F-C 5$)=4.1 \mathrm{~Hz}) ; 113.86\left(\mathrm{~d}, \mathrm{C}-6, J\left(\mathrm{~F}-\mathrm{C}^{6}\right)=3.7\right.$ $\mathrm{Hz}) ; 107.40\left(\mathrm{~d}, \mathrm{C}-2, J\left(\mathrm{~F}-\mathrm{C}^{2}\right)=26.1 \mathrm{~Hz}\right) ; 72.95(\mathrm{C}-13) ; 62.60(\mathrm{C}-$ 15); 46.39(C-14); 66.91(C-8, C-10); 50.97(d, C-7, C-11, J(F$\left.\mathrm{C}^{7,11}\right)=2.9 \mathrm{~Hz}$ ). Elemental Analyses: Calculated for $\mathrm{C}_{15} \mathrm{H}_{\mathrm{FN}} \mathrm{O}_{2}: \mathrm{C}: 61.21 \% \mathrm{H}: 6.51 \% \mathrm{~N}: 9.52 \%$. Found: C : C : $61.02 \%$ H: $6.70 \%$ N: $9.50 \%$.

Biological Assays: The oxazolidinone derivatives were evaluated for in vitro activity by determining minimum inhibitory concentration against $S$. aureus ATCC 6538 by agar dilution method [8].

Molecular mechanics calculations: Molecular, topological, conformational characteristics on 3D oxazolidinone derivatives optimized structure were calculated using Spartan 14 Software [10]

Docking studies: Molecular docking approach, using CLC Drug Discovery Workbench Softw are was conducted in order to achieve accurate predictions on optimized conformation for both, the oxazolidinone compounds (as ligand) and their target receptor protein (Staphylococcus Aureus ribosomal subunit, PDB ID: 4WFA) to form a stable complex.

\section{Results and discussions}

It were obtained some oxazolidinone compounds (Scheme 1). 3,4-Dfluoro-nitrobenzene with excess piperidine or morpholine selectively gave the $p$-substituted nitrobenzene (3). Reduction of (3) compounds was followed by attachment of a carbobenzoxy activating group to the arylamines (4). Carbamate (5) was deprotonated with $n$-Bu-Li and then (R)-glycidyl butyrate was added to give the alchools (6).The oxazolidinone compounds was characterized structurally and 


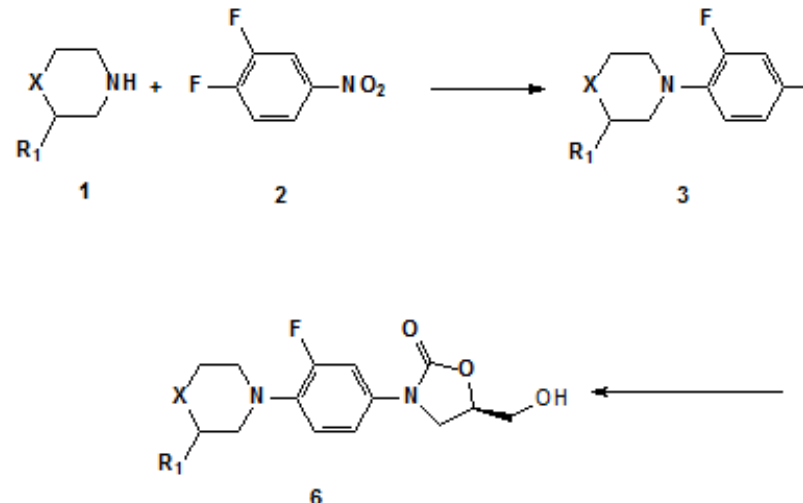

pharmacologically. All compounds exhibit moderate activity from Staphylococcus aureus, MIC > $64 \mu \mathrm{g} / \mathrm{mL}$.

Ligand preparation: The ligands have been prepared using SPARTAN'14 software package [10]. In this study, the DFT/B3LYP/6-31 G*level of basis set has been used for the computation of molecular structure, vibrational<smiles>[R3]C1CCN(c2ccc(N)cc2F)CC1</smiles>

Scheme 1. Synthesis of oxazolidinone compounds

\begin{tabular}{|c|c|c|c|c|c|c|c|c|c|c|}
\hline \multirow[b]{2}{*}{$\begin{array}{l}\text { ¿ } \\
\text { 兽 } \\
\text { 兽 }\end{array}$} & \multicolumn{10}{|c|}{ Molecular properties } \\
\hline & 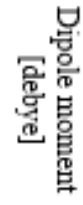 & 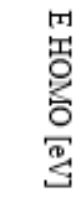 & 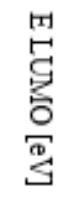 & 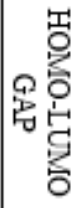 & 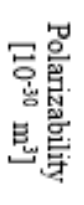 & $\begin{array}{l}\text { 恕 } \\
\text { 点 } \\
\text { 总 }\end{array}$ & $\begin{array}{l}\text { 였 } \\
\text { 窇 }\end{array}$ & 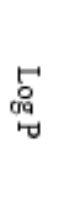 & 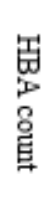 & 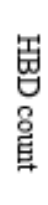 \\
\hline Alcohol 1 & 2.82 & -5.44 & -0.28 & 5.72 & 62.87 & 50.368 & 1.44 & 1.03 & 5 & 1 \\
\hline Alcohol3 & 3.21 & -5.29 & -0.16 & 5.45 & 63.61 & 42.451 & 1.45 & 2.16 & 4 & 1 \\
\hline Alcohol 4 & 3.15 & -5.27 & -0.14 & 5.41 & 65.08 & 42.482 & 1.48 & 2.56 & 4 & 1 \\
\hline Alcohol 5 & 3.18 & -5.29 & -0.16 & 5.45 & 65.08 & 42.472 & 1.48 & 2.49 & 4 & 1 \\
\hline
\end{tabular}

Table 1

MOLECULAR PROPERTIES FOR CPK MODEL COMPUTATIONS FOR OXAZOLIDINONE COMPOUNDS USING SPARTAN 14 V1.1.4 SOFTWARE. a

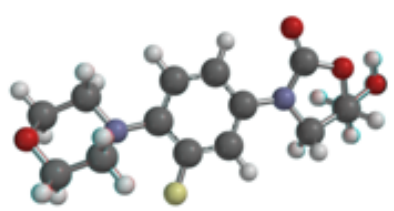

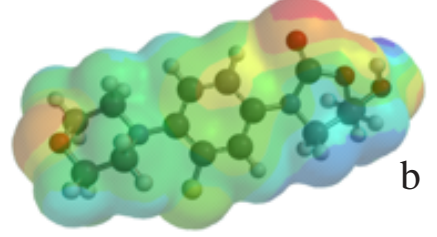

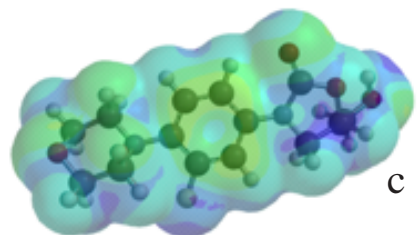

Fig. 2. The optimized geometry (2a), electrostatic potential pattern of the surface of Alcohol 1 (red- negative, high electron density, bluepositive area, low electron density) (2b) and local ionization potential map (2c)

a

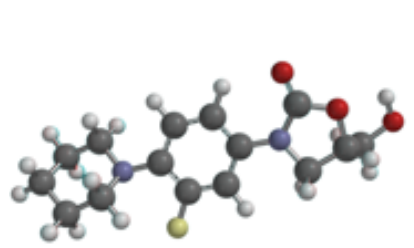

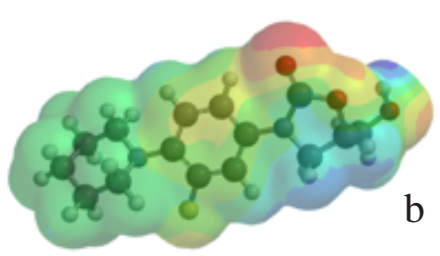

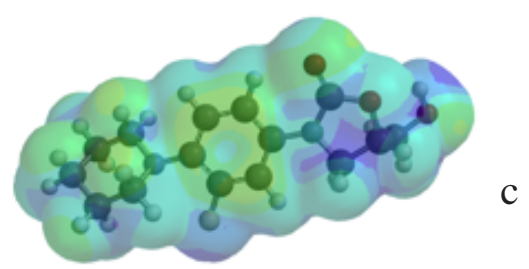

C

Fig. 3. The optimized geometry (3a), electrostatic potential pattern of the surface of Alcohol 3 (red- negative, high electron density, bluepositive area, low electron density) (3b) and local ionization potential map (3c)

a

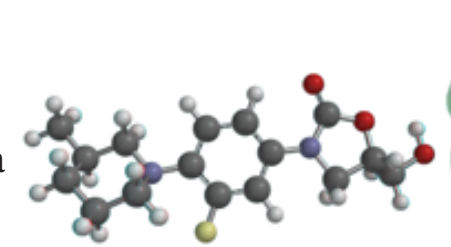

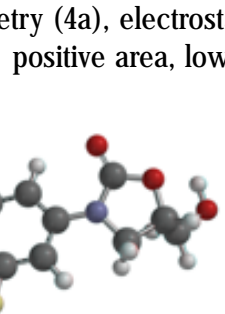

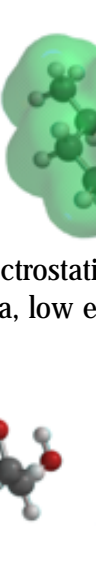

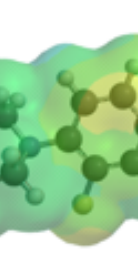

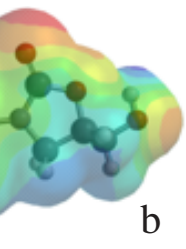

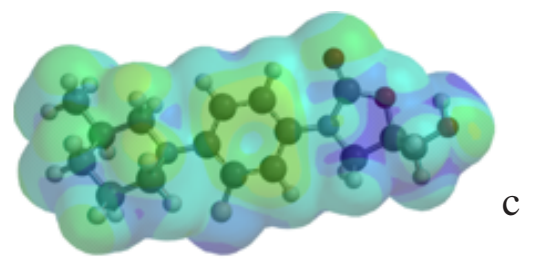

Fig. 4. The optimized geometry (4a), electrostatic potential pattern of the surface of Alcohol 4 (red- negative, high electron density, blue-
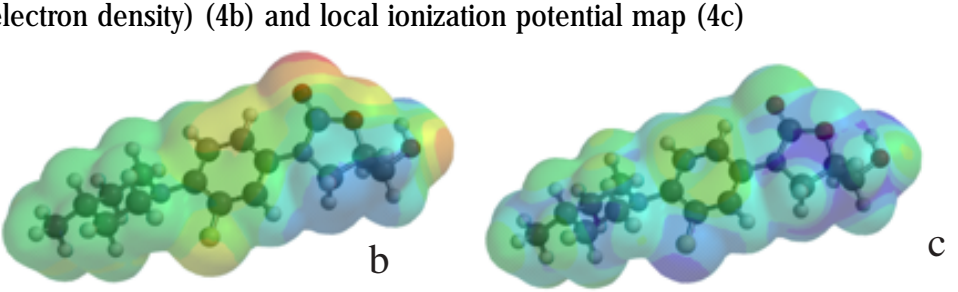

Fig. 5. The optimized geometry (5a), electrostatic potential pattern of the surface of Alcohol 5 (red- negative, high electron density, bluepositive area, low electron density) (5b) and local ionization potential map (5c) 
Table 2

THE LIST OF INTERMOLECULAR INTERACTIONS BETWEEN THE LIGAND OXAZOLIDINONES DOCKED WITH 4WFA

\begin{tabular}{|c|c|c|c|c|}
\hline Comp. & $\begin{array}{l}\text { Score/ } \\
\text { RMSD }\end{array}$ & Interacting group & Hydrogen bond & $\begin{array}{l}\text { Length } \\
\text { bond }\end{array}$ \\
\hline $\begin{array}{l}\text { LZD co- } \\
\text { crystallized }\end{array}$ & $\begin{array}{c}-32.73 / \\
1.88\end{array}$ & $\begin{array}{l}\text { ARG 33:I, GLY 34:I, HIS 35:I, LYS 36:I, GLY } \\
\text { 37:I, GLN 38:I, LYS 39:I, ARG 41:I, } \\
\text { ALA 40:I, SER 42:I }\end{array}$ & $\mathrm{N} \mathrm{sp}^{2}(\mathrm{~N} 4)-0 \mathrm{sp}^{2} \mathrm{din}$ GLN $38: \mathrm{I}$ & $2.989 \mathrm{~A}$ \\
\hline Alcohol 1 & $\begin{array}{c}-35.52 \\
0.08\end{array}$ & $\begin{array}{l}\text { LYS 39:I, ALA 40:I, GLN 38:I, HIS 35:I, LYS } \\
\text { 36:I, SER 42:I, ARG 41:I }\end{array}$ & 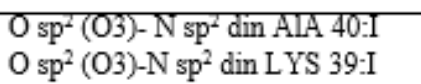 & $\begin{array}{l}2.585 \mathrm{~A} \\
3.085 \AA\end{array}$ \\
\hline Alcohol3 & $\begin{array}{c}-32.74 \\
0.53\end{array}$ & $\begin{array}{l}\text { LYS 39:I, ALA 40:I, GLN 38:I, HIS 35:I, LYS } \\
\text { 36:I, SER 42:I, ARG 41:I, GLY 34:I }\end{array}$ & 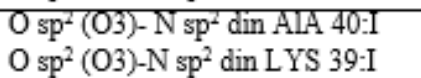 & $\begin{array}{l}2.578 \mathrm{~A} \\
3.028 \AA\end{array}$ \\
\hline Alcohol 4 & $\begin{array}{c}-37.86 \\
0.08\end{array}$ & $\begin{array}{l}\text { LYS 39:I, ALA 40:I, GLN 38:I, HIS 35:I, LYS } \\
\text { 36:I, SER 42:I, ARG 41:I, GLY 34:I }\end{array}$ & $O \mathrm{sp}^{3}\left(074-\mathrm{N} \mathrm{sp}^{2}\right.$ din LYS 39-I & $2.948 \mathrm{~A}$ \\
\hline Alcohol5 & $\begin{array}{c}-38.11 / \\
0.06\end{array}$ & $\begin{array}{l}\text { LYS 39:I, ALA 40:I, GLN 38:I, HIS 35:I, LYS } \\
\text { 36:I, SER 42:I, ARG 41:I. }\end{array}$ & $\begin{array}{l}O \mathrm{sp}^{2}(\mathrm{O} 3)-\mathrm{N} \mathrm{sp}^{2} \operatorname{din} \text { AIA } 40: \mathrm{I} \\
\mathrm{Osp^{2 }}(\mathrm{O} 3)-\mathrm{N} \mathrm{sp}^{2} \operatorname{din} \mathrm{L} Y \mathrm{~S} 39-\mathrm{I}\end{array}$ & $\begin{array}{l}2.635 \mathrm{~A} \\
3.105 \mathrm{~A}\end{array}$ \\
\hline
\end{tabular}

molecular dipole moment, polar surface area (PSA), the ovality, polarizability, the octanol water partition coefficient $(\log P)$, the number of hydrogen-bond donors (HBDs) and acceptors (HBAs). The polarizability is useful to predict the interactions between non-polar atoms or groups and other electrically charged species, such as ions and polar molecules having a strong dipole moment.

\section{Compounds molecular properties}

Molecular Docking:The steps to go through to explore protein-ligand interaction using docking, are: setup the binding site in a Molecule Project, dock ligands imported to a Molecule Table, inspectthe docking results.The docking studies have been carried out using CLC Drug Discovery Workbench Software. The score and hydrogen bonds formed with the amino acids from group interaction atoms are used to predict the binding modes, the binding affinities and the orientation of the docked compounds (fig. 6) in the active site of the protein-receptor (table 2). It was realized molecular docking studies in order to to identify and visualize the most likely interaction, the binding affinities and the orientation of the docked ligands at the active site of Staphylococcus aureus ribosomal subunit (PDB ID: 4WFA) [11].

\section{Docking method validation}

The ensure that the ligand orientations and position obtained from the molecular docking studies are valid and reasonable potential binding modes of ligands, the docking methods and parameters used have been validated by redocking (fig. 7).

\section{Calculate molecular properties}

Using the Calculate Molecular Properties tool it have been calculated commonly used properties of small molecules, such as Lipinski's rule of five [12]: number of hydrogen bond donors less than 5 (the total number of nitrogen-hydrogen and oxygen-hydrogen bonds), number of hydrogen bond acceptors less than 10 (the total number of nitrogen and oxygen atoms), the molecular weight less than 500 Daltons; Log P (octanol-water partition coefficient) less than 5 . The calculation of the $\log P$ is based on the XLOGP3-AA method [13].The number of violations of the Lipinski rules gives an indication of how drug-like for a molecule is. In general, orally active drugs have fewer than two violations. These properties can be useful for identifying potential drug-like molecules, or for removing non drug-like molecules from a compound library before starting a large virtual screening experiment (table 3).

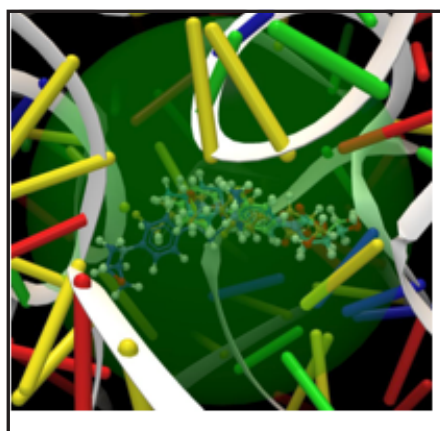

Binding site

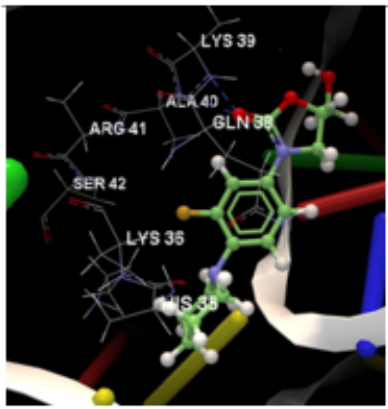

Interacting group of Alcohol 3

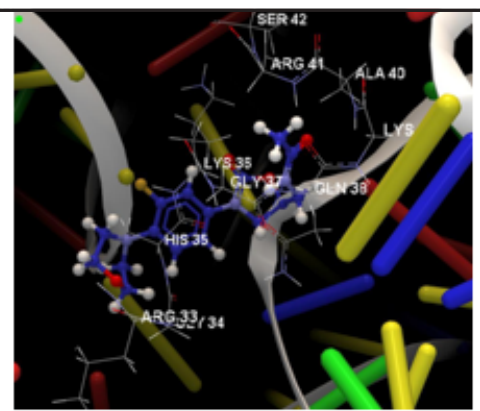

Interacting group of LZD cocrystallized

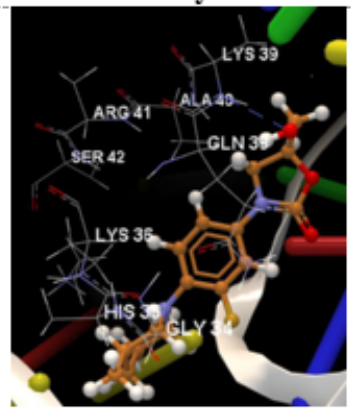

Interacting group of Alcohol 4

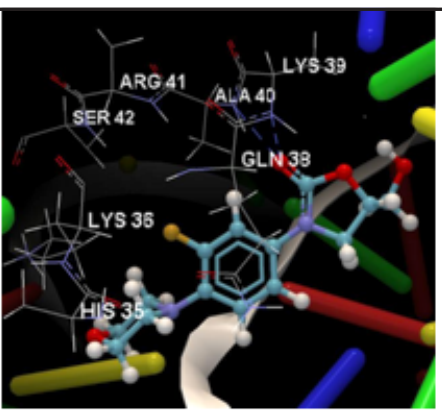

Interacting group of Alchool 1

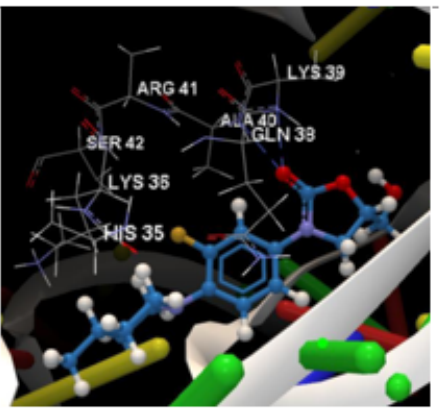

Interacting group of Alcohol 5
Fig. 6. The binding affinities and the orientation of the docked compound 


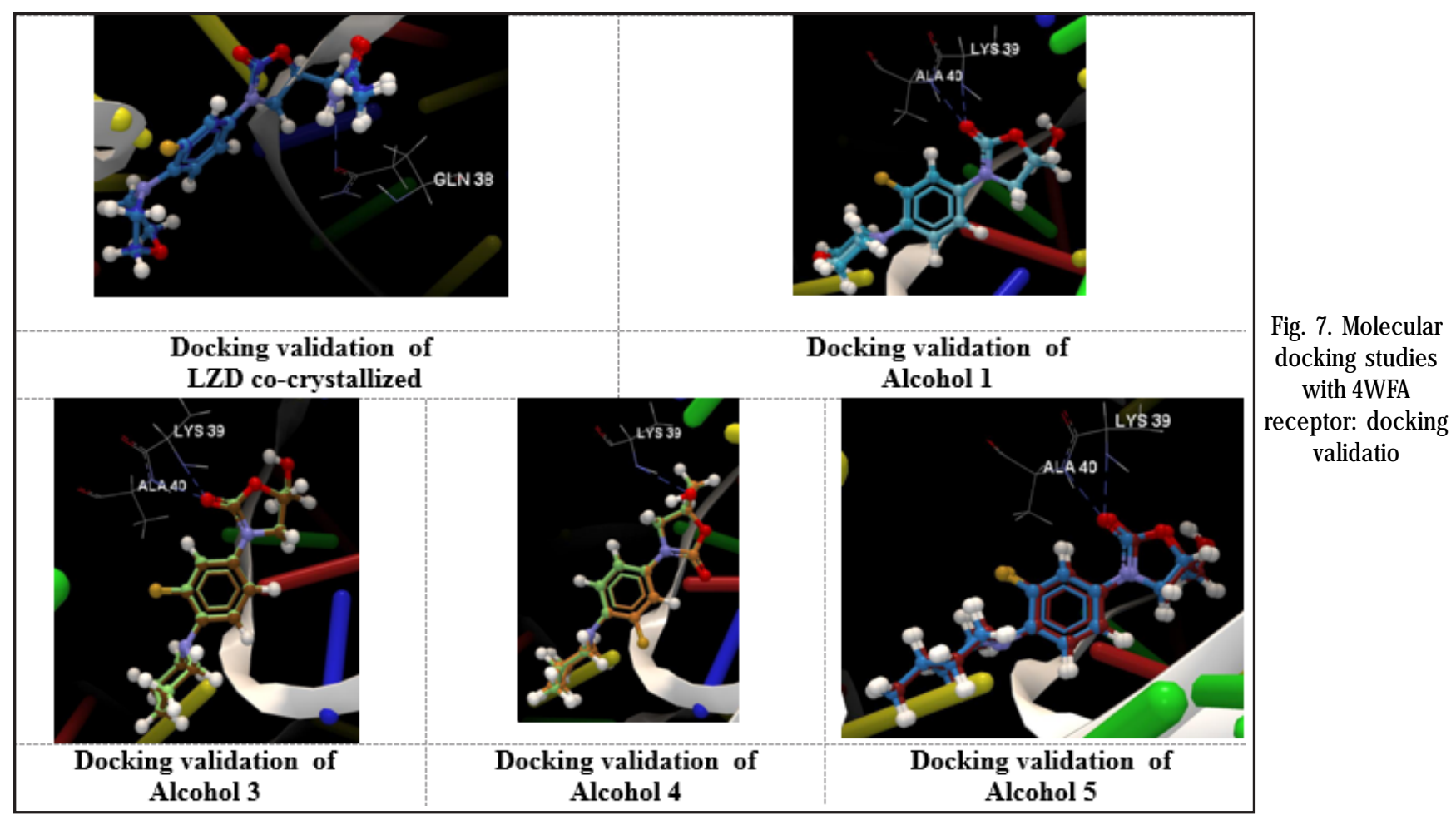

\begin{tabular}{|l|c|c|c|c|c|c|c|}
\hline Compound & Atoms & $\begin{array}{c}\text { Weight } \\
\text { [Daltons] }\end{array}$ & $\begin{array}{c}\text { Flexible } \\
\text { bonds }\end{array}$ & $\begin{array}{c}\text { Lipinski } \\
\text { violations }\end{array}$ & $\begin{array}{c}\text { Hydrogen } \\
\text { donors }\end{array}$ & $\begin{array}{c}\text { Hydrogen } \\
\text { acceptors }\end{array}$ & LogP \\
\hline Alcohol 1 & 38 & 296.29 & 3 & 0 & 1 & 6 & 0.69 \\
\hline Alcohol 3 & 40 & 294.32 & 3 & 0 & 1 & 5 & 1.91 \\
\hline Alcohol 4 & 43 & 308.35 & 3 & 0 & 1 & 5 & 2.34 \\
\hline Alcohol 5 & 43 & 308.35 & 3 & 0 & 1 & 5 & 2.34 \\
\hline
\end{tabular}

Table 3

LIGANDSWITH PROPERTIES

\section{Drug-likeness of the oxazolidinone compounds}

According to the data presented in table 3, all oxazolidinone compounds have zero violation of all the parameters involved in Lipinski's rule of five.

\section{Conclusions}

We have synthesized somme oxazolidinone compounds and we have investigated their antibacterial activity. For the synthesized oxazolidinone derivatives, a study of the characteristics and molecular properties has been realized. The docking studies revealed that the all compounds showed good docking score. The docking score is a measure of the antimicrobial activity of the studied compounds.

Acknowledgements: This paper has been financed through the NUCLEU Program, which is implemented with the support of ANCSI, Project no. PN 16-27 0101

\section{References}

1.BARBACHYN MR, HUTCHINSON DK, BRICKNER SJ, CYNAMON MH, KILBURN JO, KLEMENS SP, GLIKMAN S.Z., GREGA KC, HENDGES SK, TOOPS DS, FORD CW, and ZURENKO GE., J.Med. Chem.1996; 38,680685.

2.BRICKNER SJ , BARBACHYN MR, HUTCHINSON DH and MANNINEN PR, J.Med. Chem. 2008; 51, 1981-90.
3.BRICKNER SJ, DOUGLAS KH, BARBACHYN MR, MANNINEN PR, ULANOWICZ DA, GARMON SA, GREGA KC, HENDGES SK,TOOPS DS, FORD CW, and ZURENKO GE, J.Med. Chem.1996; 39,673-679.

4.EYAL Z, MATZOV D, KRUPKIN M, WEKSELMAN I, PAUKNER S, ZIMMERMAN E, ROZENBERG $H$, BASHAN A, YONATH A, Proc.Natl.Acad.Sci.USA, 2015; 112: E5805-E5814.

5. W.ALSH CT and WENCEWICZ TA; The Journal of Antibiotics, 2014, 67,7-22.

6.MIKHAIL FG and ZHENGYU YY, J.Med. Chem. 2014, 57,4487-4497;

7.MICHALSKA K, KARPIUK I, KROL M, TYSKI T, Bioorg, Med. Chem. 2013, 21, (3), 577-591

8.PINTILIE, L, STEFANIU, A, NICU, A I, CAPROIU, M.T., MAGANU, M., Rev. Chim. (Bucharest), 67, no. 3, 2016, p. 438-445

9.PINTILIE L, DEACONU M, STEFANIU A, TANASE C, CAPROIU MT, Rev. Chim.(Bucharest), 67, no. 4, 2016, p. 613-620 10.SPARTAN'14 WAVEFUNCTION, INC. IRVINE, CA

11.EYAL Z, MATZOV D, KRUPKIN M, WEKSELMAN I, PAUKNER S, ZIMMERMAN E, ROZENBERG $H$, BASHAN A, YONATH A, , Proc.Natl.Acad.Sci.USA, 2015; 112: E5805-E5814,

12.LIPINSKI CA, LOMBARDO F, DOMINY B W AND FEENEY $P$ J..Advanced Drug Delivery Reviews.2001;46:3-26;

13.CHENG T, ZHAO Y, LI X, LIN F, XU Y, ZHANG X, LI Y AND WANG R.. J. Chem. Inf. Model. 2007;47:2140-2148.

Manuscript received: 14.12 .2017 